\section{Localisation and management of respiratory distress}

\section{Lynelle Johnson}

Assessment of the pattern of breathing, careful examination and auscultation of the respiratory tract, and swift determination of the history of the complaint will assist in determining the site responsible for generation of respiratory distress. Respiratory difficulty associated with extrathoracic airway disease typically results in respiratory difficulty on inspiration. Prolonged respiration is expected and is almost always present during inspiration but can also be detected on expiration when a large airway is obstructed. Loud breathing sounds are anticipated. Additional clinical complaints might include voice change, gagging, retching or cough. Diagnostic investigation should include cervical as well as thoracic radiographs; cervical ultrasonography might also be useful. Respiratory distress is often alleviated by supplementation with oxygen and judicious use of sedatives. If laryngoscopy is indicated, prepare for challenging intubation, tracheostomy or surgical intervention.

Expiratory effort can also indicate disease of small airways associated with inflammation (chronic bronchitis or asthma) or bronchomalacia. Cough generally predominates in these cases and expiratory prolongation can be evident. In cats, active bronchoconstriction can be reversed with administration of terbutaline, but this drug is less effective in cats with chronic bronchitis and unlikely to be beneficial in bronchomalacia. In general, dogs do not bronchoconstrict with various respiratory diseases and terbutaline is less effective.

Parenchymal and pleural diseases usually result in rapid breathing and mixed inspiratory-expiratory effort or paradoxical breathing can be encountered. Lung sounds are generally increased or harsh with parenchymal diseases such as pneumonia and fibrosis, and adventitious lung sounds (crackles) are sometimes heard. In contrast, pleural disease is characterised by an absence of lung sounds. Fluid gravitates ventrally and dampens both heart and lung sounds, while air will rise dorsally, causing an absence of lung sounds in that region. In the animal with severe respiratory difficulty, placement in an oxygen-enriched environment is recommended to stabilise the animal and reduce stress. If lung sounds are absent, thoracocentesis is advised and, depending on the animal's demeanour, sedation may or may not be beneficial. Radiographs are performed when the animal's breathing has improved.

\section{KEY LEARNING OBJECTIVES}

- Generate a list of differential diagnoses for the dog or cat with inspiratory respiratory difficulty

- Compare auscultation findings in animals with parenchymal versus pleural disease

- Create a rational therapeutic plan for the animal in respiratory distress, based on the most likely localisation of disease

\section{MULTIPLE CHOICE QUESTIONS}

1. What clinical sign or physical examination finding is NOT associated with laryngeal paralysis?
a. Voice change
b. Lung hyperinflation
c. Inspiratory respiratory distress
d. Stridor

2. Loss of negative pressure due to pneumothorax results in all of the following except what?
a. Lung collapse
b. Increased venous return
c. Decreased cardiac output
d. Rapid breathing

3. Which of the following is the least likely differential diagnosis in a cat presented for evaluation of cough and difficulty breathing?
a. Asthma/bronchitis
b. Congestive heart failure
c. Mycoplasma pneumonia
d. Primary pulmonary neoplasia

4. Which of the following is NOT a recognised cause of chylothorax?
a. Heartworm disease
b. Lung lobe torsion
c. Feline infectious peritonitis (FIP)
d. Neoplasia

5. Which of the following does an animal with pleural effusion demonstrate?
a. Marked inspiratory effort
b. Loss of lung sounds ventrally
c. Severe tracheal sensitivity
d. Slow, deep breathing

\section{Respiratory disease in rabbits: not just Pasteurella}

\section{Yvonne van Zeeland}

In rabbits, respiratory disease has commonly been attributed to infections with Pasteurella. Indeed, this pathogen is commonly isolated from the respiratory tract in rabbits. Nevertheless, various other pathogenic organisms, including Bordetella, Pseudomonas and Staphylococcus spp., may be isolated as well. Thus, although Pasteurella may certainly be considered an important cause of respiratory disease in rabbits, other causes should also be taken into consideration and included within the differential diagnoses of rabbits with dyspnoea.

Upon evaluation of respiratory disease, different types of classification may be used, which can be based on aetiology, type and pattern of the associated signs and symptoms, or the location, organ or tissue involved in the disease. 